\title{
Review: Chronopharmacology; A Biological Rhythm
}

\author{
Pooja P. Dahale ${ }^{*}{ }^{1}$, Vijay R. Chakote ${ }^{2}$, Siddesh V Rokade ${ }^{3}$, Shrikant C. Bhosale ${ }^{4}$
}

\section{Department of Pharmaceutics}

Mr. Vijay R. Chakote ${ }^{2}$, SVERI College of pharmacy, Pandharpur, Maharashtra, India

\section{B.Pharmacy Final year Students}

Siddesh V Rokade ${ }^{3}$, Shrikant C. Bhosale ${ }^{4}$, Indrayani Institute of Pharmaceutical Education \&

Research, Talegaon dabhade, Pune

Corresponding author

Pooja P. Dahale

Lecturer, MCP, Nilanga, Latur Maharashtra

EmailId:vijayrchakote@gmail.com

ContactNo.9970747432 


\section{Introduction;}

Chronopharmacology is the study of how the effects of drugs vary with biological timing and endogenous periodicities.

The goal is to improve our understanding of periodic and thus predictable (e.g. circadian) changes in both desired effects (chronoeffectiveness) and tolerance (chronotolerance) of medications. Dosing time-dependent changes also include quantification of parameters characterizing endogenous circadian rhythms (CR), in terms of pharmacologic effects, e.g. the 24-h adjusted mean (M), the period, the amplitude (A, the peak-to-trough difference), and the acrophase, the peak time location in the 24-h scale). Chronopharmacology became recognized as a scientific domain of investigation only in the early 1970s. For conventionally trained pharmacologists, it was not clear that predictable temporal variations of effects and disposition of agents (e.g. medications, hormones, and toxic substances) are governed by endogenous biological rhythms rather than by changes of external factors. On the 24 -h scale (as well as on the yearly scale) there are peaks and troughs of physiological variables that are not randomly distributed; their respective locations correspond to a temporal organization controlled by a set of pacemakers (so-called biological clocks) became recognized as a scientific domain of investigation only in the early 1970s.

\section{CONCEPT OF CHRONOPHARMACOLOGY:}

\section{CHRONOKINETICS;}

Chronokinetics are defined as dosing time-dependent and predictable (rhythmic) changes in parameters used to characterize the pharmacokinetics (or the bioavailability) of a drug, e.g. 
maximum concentration (Cmax), span of time to reach $\mathrm{Cmax}$ (tmax), area under the concentration-time-curve (AUC), and half-life.

Chronokinetics of certain drugs may involve changes from a mono- to a multicompartmental model as a function of drug dosing time. Chronokinetics of drugs have been validated for many animal species including humans, with both acute and chronic administration even for sustained release having a half-life long as $84 \mathrm{~h}$.

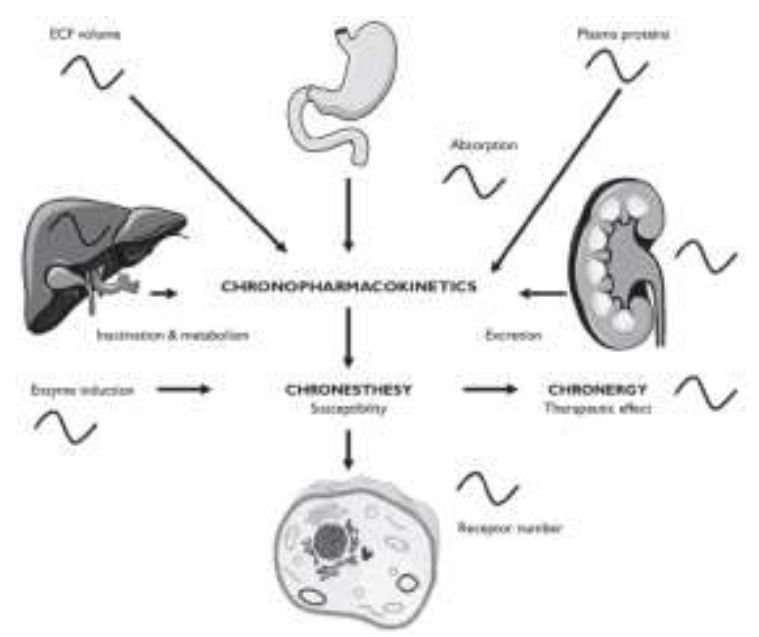

Fig; Chronokinetics

\section{$\underline{\text { 2.1 Mechanisms Underlying Chronokinetic Changes; }}$}

With related to physical properties of drugs (e.g. hydrophilicity, lipophilicity, and solubility), circadian changes in biosystems have been explored and even modulated with regard to their absorption (e.g. speed of gastric emptying and intake from gut, lung, and skin), distribution (e.g., blood flow through an organ and binding capacity of plasma proteins) metabolic rates relative to liver functions, and excretion relative to kidney functions (e.g. glomerular filtration, tubular reabsorption, and urinary $\mathrm{pH}$ ).Proper mathematical formulas have been used to calculate 
the absorption and excretion rate constants, the clearance, and the volume of distribution from pharmacokinetic data to validate dosing time-related changes.

\section{MECHANISM OF CHRONOPHARMACOLOGY;}

The basic unit of circadian timekeeping is the cell. Even in very complex organisms, most cells contain autonomous circuitry for circadian oscillations.

Generally speaking, this mechanism is comprised of negative feedback loops of transcription and translation: activation of a repressor gene results in its later repression by its own protein product, and the instability of this repressor insures this repression is short lived, so that a new cycle can begin.

- In mammals, the principal activators within this system are the CLOCK and BMAL1 proteins and their homolog, which dimerize and bind to certain elements to activate transcription of a large number of circadian genes.

- Among these circadian genes are loci encoding the PERIOD and CRYPTOCHROME families of repressor proteins (PER1-3 and CRY1-2), whose products multimerize and suppress the CLOCK.

\section{BMAL1 activating complex;}

At each of these steps, additional precision and regulatory finesse is achieved through interaction with a wide range of auxiliary proteins: kinases that phosphorylate clock proteins to modify their stability or activity. 
An example of this is seen in how evening doses of antihypertensive therapy can be used to prevent morning rises in blood pressure. The evening dose of the drug may thus be well timed with diurnal changes in blood pressure, preventing diurnal worsening of hypertension.

\section{BIOLOGICAL RHYTHM;}

\section{Chronobiology}

Science that studies the biological rhythms or the science dealing with phenomenon of rhythmicity in living organisms.

\subsection{CIRCADIAN RHYTHMS OF LIVER ENZYMES}

Radzialowski\&Bousquetdemonstrated CR in hepatic drug-metabolizing enzymes of rodents. Temporarily variations of various oxidative reactions catalyzed by the monoxygenase system have been reported for substrates such as aminopyrine,paranitroanisole, hexobarbital and 4dimethyaminobenzene, aniline, benzphetamine, benzpyrene, imipramine, etc.

The oxidative reaction was peaking in the middle of the (nocturnal) activity span. Moreover, it has also been demonstrated that $\mathrm{CR}$ of corticosterone adrenal secretion control $\mathrm{CR}$ of involved enzymes. 


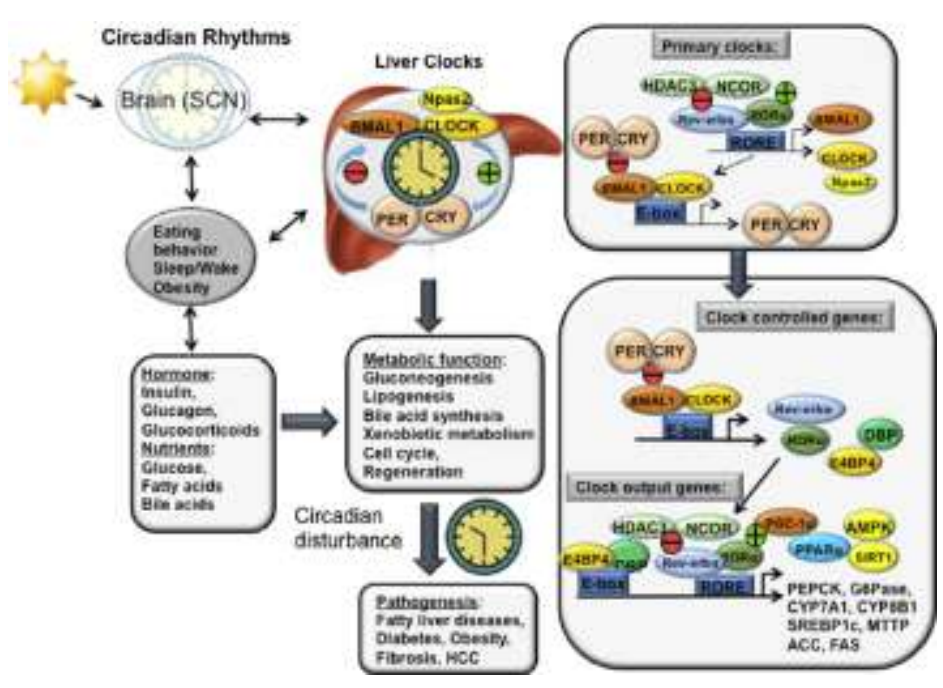

Fig; Circadian rhythms of liver

\subsection{CIRCADIAN RHYTHMS IN PLASMA PROTEIN BINDING OF}

\section{$\underline{\text { DRUGS }}$}

The phenomenon of CR in plasma protein binding of drugs was first demonstrated for cortisol and thereafter for its synthetic analogs CR in plasma total proteins, albumin, globulins, etc, related to liver activity, induces circadian changes in the (active) fraction of drugs and hormones.

.For example; A normal circadian rhythm of plasma corticosterone given as; 


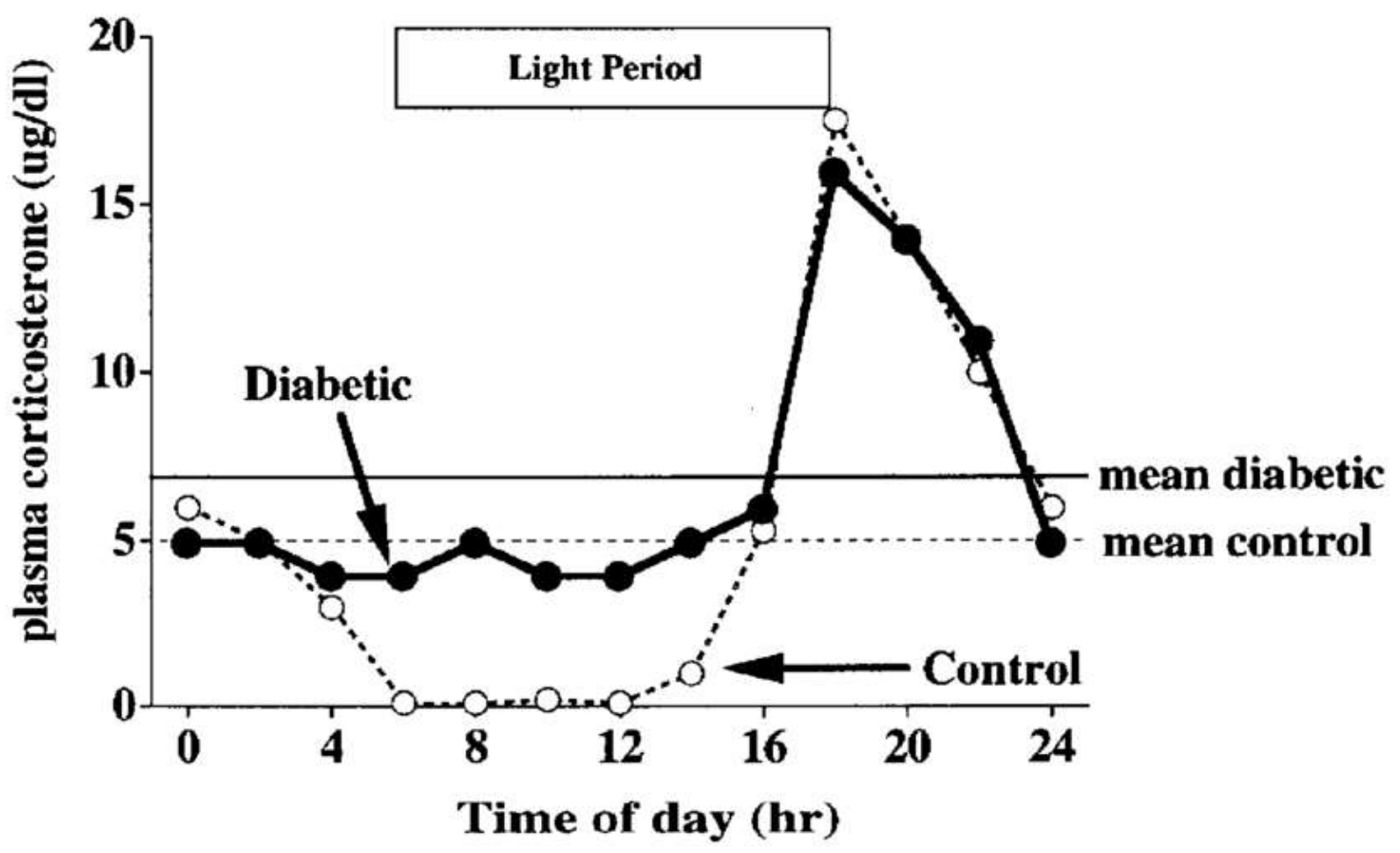

Fig; Circadian rhythm of plasma corticosterone

In the rat the peak time of protein binding occurs during the (nocturnal) activity spans for disopyramide, lidocaine, and carbamazepine which also correspond to the peak time of plasma proteins.

\subsection{Circadian Rhythms of Receptors}

CR have been documented and quantified in various receptors and organs, e.g. brain and heart in rats and blood cells in human. In all cases a CR was observed for the number of binding sites rather than for binding capacity of sites. Statistically significant 24-h rhythms occurred in all receptors studied byWirz-Justice and coworker as well as by others using homogenates from the whole rat forebrain. In addition, waveform, amplitude, and acrophase of these circadian 
changes vary with the time of year, even though rats have been kept synchronized with a defined and constant LD cycle.

These rhythms are endogenous because they persist under constant environmental conditions without a known zeitgeber and also when animals are deprived of sleep. The pattern of a receptor rhythm may change from one brain region to another.

For example, the pattern can be different from the same ligand in different nuclei of the hypothalamus. In rats, receptor rhythms vary according to strain and even within the same strain from different breeding lines. Binding to a given ligand in a defined brain region varies with age, leading to changes in CR parameters such as amplitude, acrophase shift, and/or 24-h mean.

However, the circadian amplitude in the number of certain receptors is sometimes not large enough to explain fully the impressive magnitude of an observed change in chronesthesy.

\subsection{Circadian rhythm in neuroendocrine system;}

The high-amplitude circadian rhythms in the hypothalamic-pituitary-adrenocortical (HPA) and autonomic nervous systems contribute to the day-night difference in airway caliber and risk of NBA. In diurnally active asthmatics, serum cortisol con-centration is greatest around the commencement of day time activity and lowest during the middle of the nighttime . Serum epinephrine concentration increases rapidly following morning awakening and reaches peak level by the middle of the activity period. It declines thereafter and reaches lowest concentration during sleep, when cholinergic (vagal) dominates . Cortisol exerts anti-inflammatory effect and it up-regulates $\beta$-receptor function; epinephrine induce s bronchodilation and exerts a stabilizing 
effect on the mast cellmembrane, thereby retarding the release of pro-inflammatory mediator substances

Thus, the high-amplitude circadian rhythms in cortisol and epinephrine are thought to contribute significantly the day-night variation in airway inflammation, reactivity, and caliber. Several investigations suggest that some aspects of the HPA may be abnormal in NBA. The adrenal cortex of those prone to NBA may be less responsive to corticotrophin stimulation than those mostly prone to daytime BA or in normal controls .Moreover, cortisol binding and steroid responsiveness seem tobe impaired in NBA, resulting in impaired inhibition of tissue inflammatory processes .

The hormone melatonin synthesized and circulated almost exclusively during the darkness of nighttime while asleep. Melatonin plays a central role in biological clock function. Melatonin also exerts pro-inflammatory effects, and accumulating evidence suggestions may contribute to the exacerbation of airway inflammation and consequent airway hyperreactivity in NBA patients. 


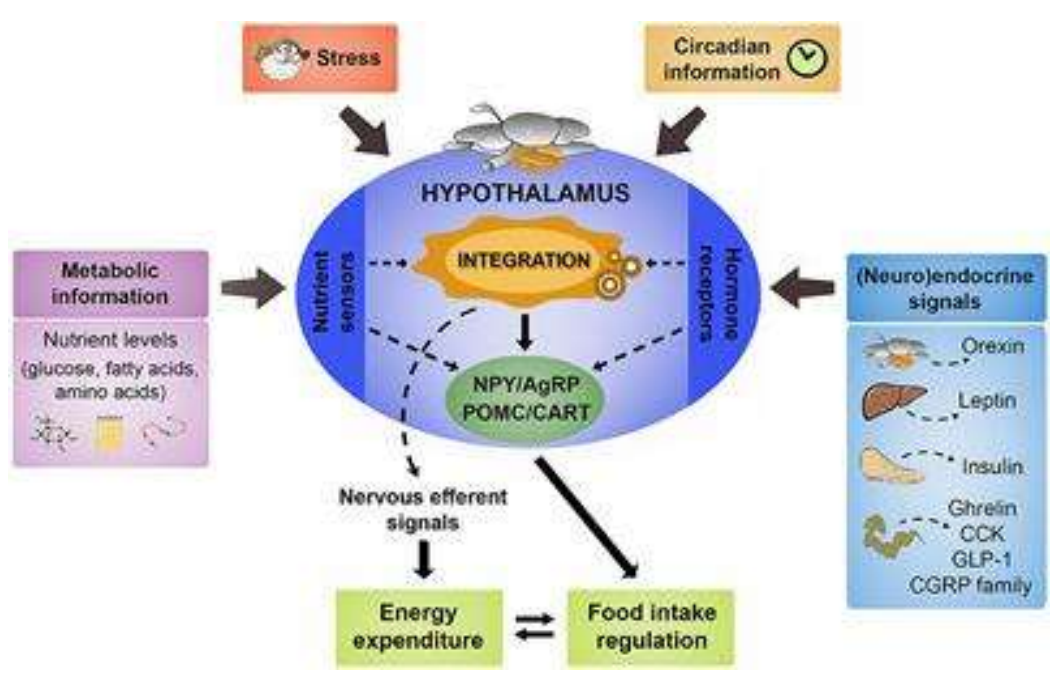

Fig; Circadian Rhythm InNeuro-Endocrine System

\section{5; Chronokinetic Changes Can Be Either Sex or Age Related, as Well as}

\section{Phenotype Related;}

In both sexes, large-amplitude CR of plasma AUC were found in studies with four different dosing times. However, both the amplitude and the 24-h mean were larger in women than in men. Dosing time-dependent changes in tmax $>C \max$, and AUC of sustained-release indomethacin (NSAID) have been demonstrated in young adults but not in elderly subjects. The polymorphism in the human potency to acetylate some drugs (e.g. isoniazid, procainamide, and hydralazine). With regard to these genetic factors, comparison between slow and rapidacetyltor types of healthy young subjects has shown statistically significant differences in the chronokinetic pattern of isoniazid.

\section{6; Circadian Rhythm in Asthma;}

Nighttime worsening of asthma has been historically recognized. A Roman physician in the fifth century, CaeliusAurelianus, noted: "On the heavy breathing and wheezing which is called 
Asthma by the Greeks, this disease is a burden during the winter and at night more than during the day or the spring .Clinically, asthma exacerbations frequently occur in the early hours of the morning, around 4:00 a.m. Dyspnea-induced nighttime awakening occurs in more than $75 \%$ of respondents in a large survey of individuals with asthma . In a 1-year review and a 2.5-year review of deaths due to asthma in adults, approximately $70 \%$ of asthma-related deaths occurred between 12:00 a.m. and 6:0 a.m.Physiologically, airway caliber and inflammation also follow circadian patterns. Peak expiratory flow (PEF) as a measure of airway obstruction has been shown to fluctuate over a 24-hour period in both healthy patients and patients with asthma. Airway obstruction worsens during the night, with patients with asthma having a $51 \%$ larger change in PEF during nighttime than control patients . In addition, Bonnet and colleagues revealed the circadian variation of airway responsiveness, with maximum bronchial responsiveness to methacholine and histamine bronchial challenge at 3:00 a.m. and 4:00 a.m. When trans bronchial biopsies were performed in patients with and without asthma at 4:00 p.m. (when lung function is optimal) and 4:00 a.m. (when airflow limitation is highest), the tissue biopsies of nocturnal patients with asthma had a pronounced circadian variation in alveolar eosinophil number per unit volume, with a significantly higher eosinophil number at 4:00 a.m. than 4:00 p.m. Broncho-alveolar lavage (BAL) studies show higher numbers of macrophages, neutrophils, lymphocytes, and CD4+ T cells have also been reported in alveolar tissue at 4:00 a.m. than at 4:00 p.m. The increase in inflammatory cells correlates with the overnight increase in airflow obstruction.

For example-Methyl prednisolone causes no suppression during a day(If given between 8:00 16:00) as shown in below figure. 


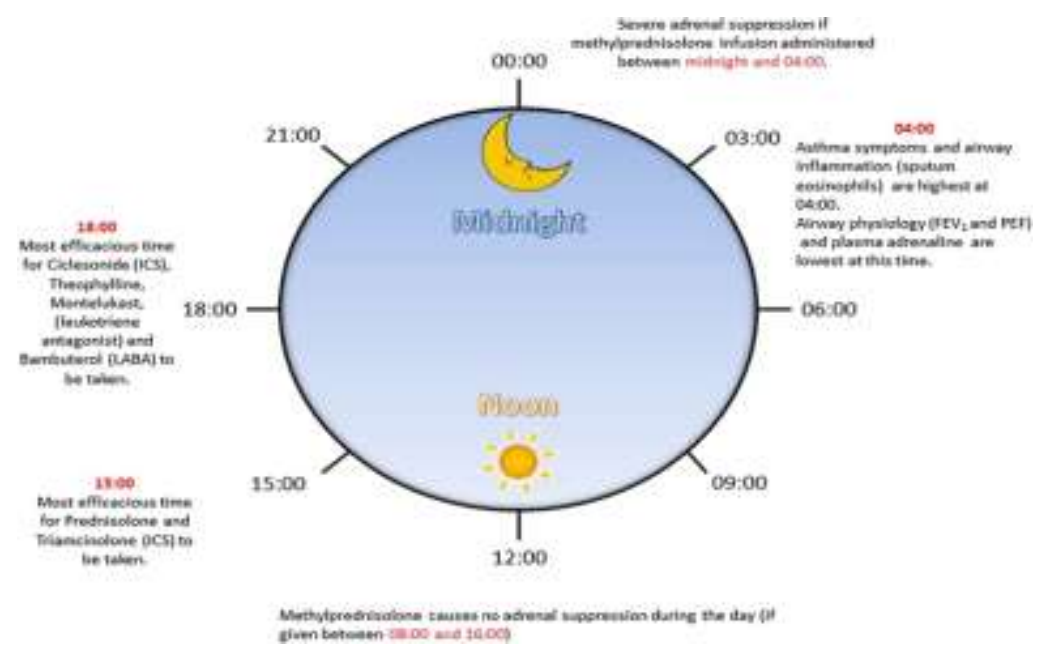

\section{Fig; Circadian Rhythm In Asthma}

\section{5; THE CIRCADIAN CLOCK}

Many biological functions wax and wane in cycles that repeat each day, month, or year. Such patterns do not reflect simply an organism's passive response to environmental changes. Rather, they reflect the organism's biological rhythms: i.e. its ability to keep track of time and to direct changes in function accordingly. Because the Earth rotates on its axis, it presents two environments, i.e. light and darkness; because the earth's axis of rotation is tilted, durations of daily periods of darkness and light vary during the course of the year.

Through evolution, animals have responded to these environmental changes by adapting to them. This is the origin of biological rhythms that repeat approximately every 24 hours, called circadian rhythms. 


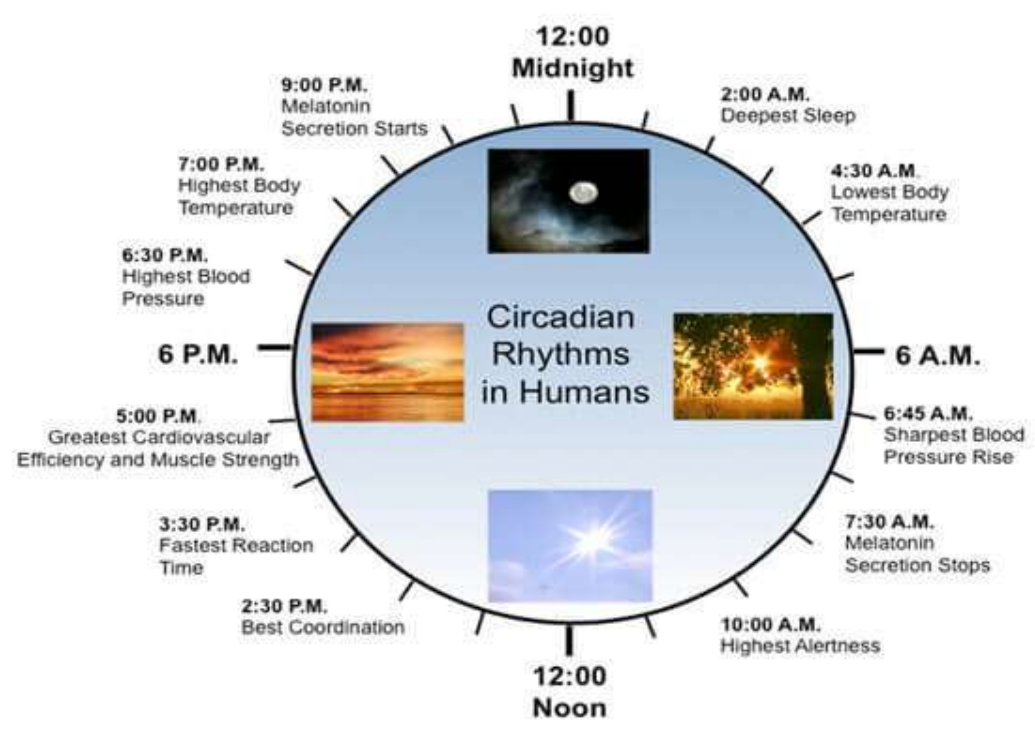

Fig; Circadian Clock- A rhythm of life

\section{THE CARDIOMYOCYTES CIRCADIAN CLOCK;}

Circadian clocks have been described in all CV-relevant cell types, including cardiomyocytes. But what might be the role of this mechanism in the heart? A general concept is that circadian clocks confer the selective advantage of anticipation, temporally partitioning biological processes in a manner that allows a cell/organ to respond appropriately to fluctuations in the environment over the 24-h day. What might the heart need to anticipate? Over the course of the day, the heart must contend with two major behavioural cycles; sleep-wake and fasting-feeding cycles.

These cycles are associated with profound fluctuations in energetic demand, neural stimulation, hormones, nutrients, oxygen tension, body temperature, and shear stress. How might the cardiomyocyte clock simultaneously anticipate oscillations in all these factors? Consistent with its transcriptional nature, circadian clocks often govern cellular functions through modulation of 
genes that are not core clock components (termed clock output genes; CCGs). Selective genetic disruption of the cardiomyocyte circadian clock indicates that this molecular mechanism regulates up to $10 \%$ of the cardiac transcriptone; known functions of CCGs in the heart span processes as diverse as signal transduction and ion homeostasis, to metabolism. The latter serves as an excellent example, illustrating the importance of temporal partitioning. Metabolic flux analysis reveals that the cardiomyocyte clock promotes oxidative metabolism at the sleepwake transition (in anticipation of increased energetic demand upon awakening), augments nutrient storage towards the end of the awake period (in anticipation of the upcoming fast during the sleep period), and increases cellular constituent turnover at the beginning of the sleep period (facilitating repair/renewal of the myocardium prior to awakening).Moreover, the myocardium exhibits clock-dependent rhythms in responsiveness to various stimuli/stresses, including adrenergic stimulation, insulin, and fatty acids. At a functional level, both time-ofday-dependent oscillations in heart rate and contractility are attenuated in mice following genetic disruption of the cardiomyocyte circadian clock; underscoring the importance of this mechanism in orchestrating critical processes in the heart, these mice develop an age-onset dilated cardiomyopathy and exhibit reduced lifespan. 


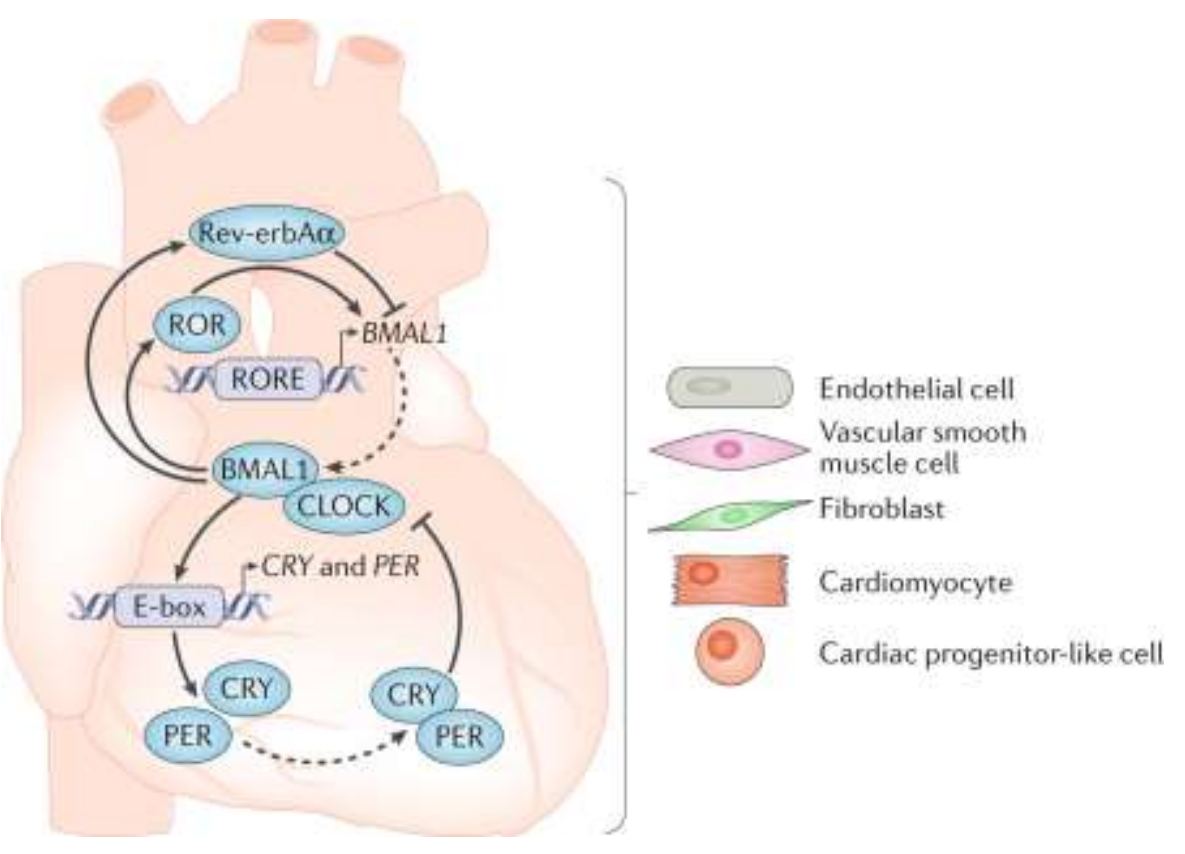

Fig; Cardiomyocytes- A Circadian Clock

\section{7; WHY THE EFFECT OF A DRUG CHANGES AS A FUNCTION OF THE TIME OF}

\section{DAY?}

An orderly timed circadian system implies that every function occurs at a specific moment in the temporal reference scale. In view of this, it is not unreasonable that xenobiotics will have differential effects as a function of the time of day.

Chronopathology describes the time-dependent effect of a pathological agent to cause the disease.

Chronopharmacology analyzes the time-dependent variations in response to a pharmacological agent. 
Chronotoxicology describes the daily changes in the activity of toxic agent on a given organism.

That the activity of any pharmaceutical agent varies as a function of time of day of administration is in contradiction to the homeostatic principle that holds that a given agent will always produce the same effect providing that a constant concentration is kept in circulation.

\section{8;RELEVANCE OF CHRONOPHARMACOLOGY IN SLEEP}

Human sleep, its duration, and organization depend on its circadian phase and, therefore, chronopharmacology is relevant in the treatment of sleep disorders. Insomnia is one of the most common of these disorders, prevalent among elderly people (40-70\% of the aged population reports sleep complaints) and causing impairment of both general physical and mental health. Nearly $30-40 \%$ of the adult population suffers from insomnia and, since it is associated with significant distress and functional decline, its treatment is advocated with top priority.

Indeed, the pharmacological treatment of insomnia has remained the most widely used treatment for decades, despite concerns about its long-term effectiveness, habituation, tolerance, and potential complications, especially in elderly people. Chronic hypnotic exposure can also carry additional risks of physical or behavioral dependence, withdrawal, rebound insomnia, and increased mortality.

\section{CIRCADIAN RHYTHM SLEEP DISORDER;}


Two components, homeostatic drive and circadian drive, interact with each other and regulate the sleep-wake cycle. The sleep-wake cycle is controlled by sleep homeostasis. The desire to sleep increases gradually with extended wakefulness and decreases during sleep.

Additionally, sleep and wakefulness occur in turn, and the timing of their occurrence is controlled by the circadian clock system.

Circadian rhythm sleep disorders (CRSDs) are defined by a persistently or recurrently disturbed sleep pattern. CRSD is attributed etiologically to alterations of the circadian timekeeping system and or a misalignment between endogenous circadian rhythm and exogenous factors that affect sleep timing . The intrinsic circadian period $(\tau$, the free-running period of circadian rhythms in the absence of external cues) is considered to be a critical factor in the pathophysiology of CRSD.

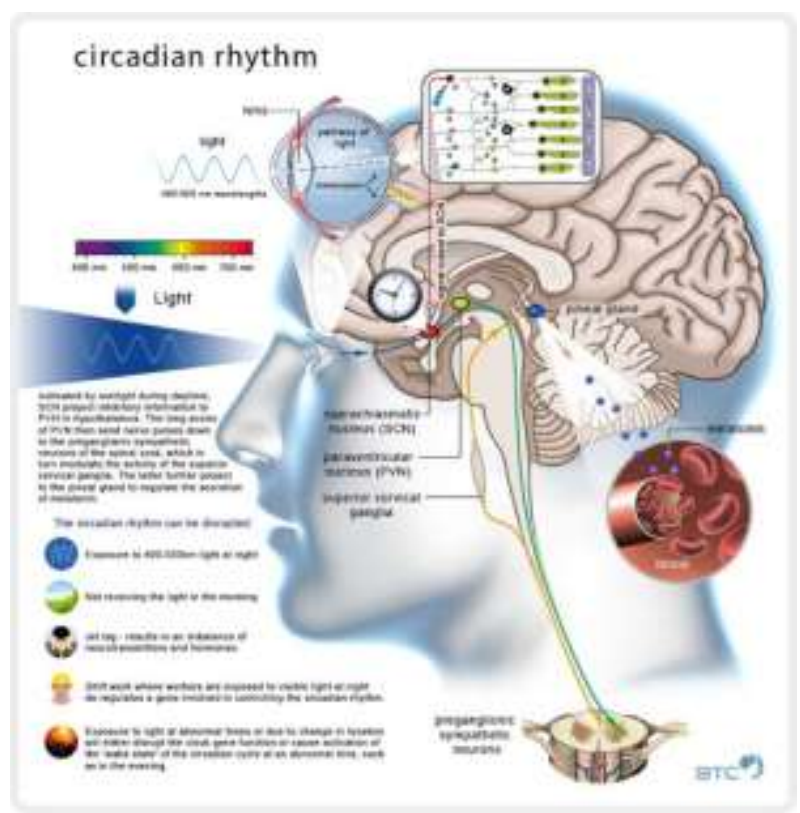

Fig ; Circadian sleep disorder 


\section{CHRONOTHERAPY;}

Biological rhythms are considered in the rational use of therapeutic agents to optimize the treatment of several diseases. One of the objectives of chronotherapy is to restore the lost temporal order found in chronic diseases. This objective implies that the dose and time of the administration of a drug should be rational and looking to reestablish the lost temporal order. Another objective is to optimize the use of a pharmacological agent so that the smallest amount possible is used at the appropriate time (highest efficacy and lowest undesired effects). For an effective chronotherapy, the results of chronotolerance and chronoefficacy of a drug must be taken into consideration. In addition, the rhythmic variations of signs and symptoms of the disease should be considered. For example, the well-known peak incidence of myocardial infarction (MI) in the morning hours has led to the timely administration of drugs such as aspirin to prevent it. However, in Southern populations with a 'siesta' habit, a second peak in MI incidence is detectable after nap time. Therefore, this should be taken into consideration when dealing with preventive interventions in these populations. It is as important to give aspirin to reduce the risk of platelet aggregation and thrombosis in the morning as in the evening to cover the two periods of susceptibility to MI. 

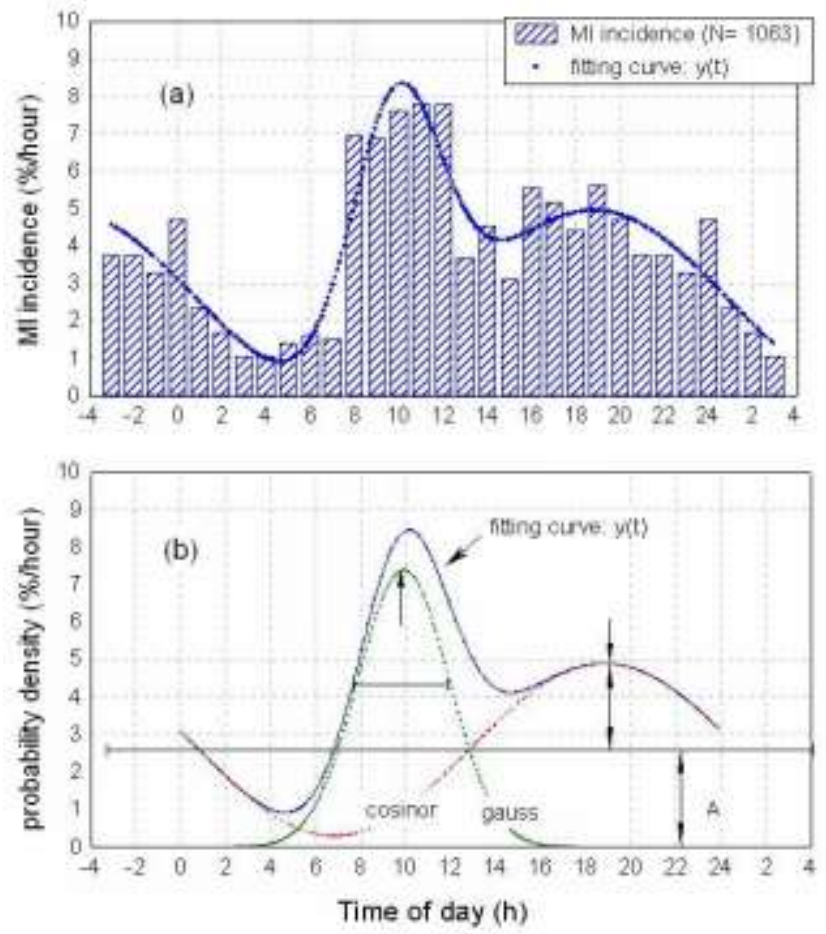

Fig; Circadian analysis of Myocardial Infaraction(MI)

\section{CHRONERGY;}

The term chronergy was introduced to designate rhythmic (temporally predictable) differences in effects of drugs on the organism as a whole Chronergy has a neutral meaning that includes rhythmic changes of both desired (effectiveness) and undesired (toxicity and its counterpart, tolerance) effects. Obviously, temporally predictable dependencies in either effectiveness or tolerance of a drug are based on both its chronokinetics and the chronesthesy of the target system. Let us consider again studies of unfractionated heparin.

The anticoagulant effect was at its trough around $08.00 \mathrm{~h}$ and at its peak around the middle of the nocturnal rest span, with a circadian amplitude of $40 \%$. The chronokinetics of the drug were documented by Decousus et al, using labeled heparin. A statistically significant CR in plasma 
clearance was observed, with a peak at $14.00 \mathrm{~h}$ and a trough at $08.00 \mathrm{~h}$ and a rather small amplitude with regard to that of the anticoagulant effect. Therefore, chronokinetic changes are not likely to explain observed CR in anticoagulant effects.

Further studies have shown time dependencies in effects of heparin in vitro, e.g. coagulation tests between blood specimens sampled at $10.00 \mathrm{~h}$ and at midnight from the same subjects. Statistically significant differences were observed in APTT and TT with greater anticoagulant effect at midnight than at $10.00 \mathrm{~h}$.

In this case, as in many others, the chronesthesy of the target system rather than the chronokinetics of the drug appears to be the key phenomenon.

\section{CHRONESTHESY;}

The term chronesthesy was used first to designate rhythmic (temporally predictable) changes in the susceptibility or the sensitivity of a target system to a drug, which cannot be explained by chronokinetic changes. Later on, taking into account many experimental findings, we proposed, with Labrecque and Smolensky thatchronesthesy be considered the chronopharmacologic counterpart of the pharmacodynamic concept. Apart from the fact that chronopharmacodynamics is a rather cumbersome and long word, it deals basically with mechanisms of time-related variation in effects and metabolism of drugs in healthy organisms. Since metabolic processes have already been taken into account when referring to chronokinetics, chronesthesy, chronoeffectiveness, and chronotolerance, the term chronopharmacodynamics does not add further precision or specification. It can be used, however, if it helps understand the meaning of chronesthesy. 
Therefore, chronokinetics and chronesthesy are complementary concepts andchronesthesy itself designates circadian and other rhythmic changes in the susceptibility of a target system. In human subjects the target can be a tissue, e.g. the skin (CR in epidermal skin reaction to intradermal injection of histamine with a nocturnal peak), the bronchial tree (CR of the bronchial response to acetylcholine as well as beta-agonists among other agents, inhaled in the form of aerosol), or the stomach (number of mucosal lesions quantified by fibroscopy is twice greater after morning [10.00 h] than evening [20.00 h] ingestion of $1 \mathrm{~g}$ of acetylsalicylic acid) . Experiments in laboratory rodents have also shown that the target biosystem may be located either at the molecular level of the receptors or in different subcellular systems (e.g. membrane structure and enzyme systems).

\section{CONCLUSION;}

The widespread awareness of the importance of the time of day on pharmacology can readily be illustrated.

The influence of the time of day on drug efficacy and toxicity is not surprising considering that most mammalian physiology is affected by the circadian clock. Currently, most approaches consider the relevance of dosing time for drug effects to recommend a standard best time for drug administration in populations with well-synchronized circadian physiology. Recently acquired knowledge on the circadian timing system and the availability of new experimental and computational models and new delivery technologies allows for the identification of the key clock and clock-controlled components that influence the dynamics of drug effects. 


\section{REFERENCES;}

1.Ko CH, Takahashi JS. Molecular components of the mammalian circadian clock. Hum Genet 2006.

2.Levi F, Schibler U. Circadian rhythms: mechanisms and therapeutic implications. Annu Rev PharmacolToxicol.

3.Dardente H, Cermakian N. Molecular circadian rhythms in central and peripheral clocks in mammals.

4.Reinberg, A., Halberg, F. 1971. Circadian chronophapharmacology.Annu. Rev. Pharmacol.

5.Radzialowski, F. M., Bousquet, W. F. 1968. Daily rhythmic variations in hepatic drug metabolism in the rat and mouse.

6..Pharmacol. Exp. Ther. 163:229-38 3.Moore-Ede. M. C.•Sulzman. F. M., Fuller, C A. 1982. The Clocks That Time Us. Cambridge, MA: Harvard Univ. Press. 448 pp.

7.Aschoff, J., Wever, R. 1981. The circadian system of man.In Handbook of Behavioral Neurobiology, ed. J. Aschoff, 4:311-48. New York: Plenum. 488 pp

8. Belanger, P. M., Lalande, M., Labrecque, G., Dore, F. 1985. Diurnal variations in the transferase and hydrolases involved in glucuronide and sulfate conjugation of rat liver. Drug Metabolism .

9. Lemmer, B., Biirmeier, H., Schmidt, S., Lang, P. H. 1987. On the daily variation in the betarccptor-adenylatecyclase-phosphodiesterase system in rat forebrain.Chronobiology. 
10. Petit, E., Milano, G., Levi, F., Thyss, A., Bailleul, F., Schneider, M. 1988. Circadianvarying plasma concentration of 5-fluorouracil during 5-day continuous venous infusion at constant rate in cancer patients. 